International Mathematical Forum, Vol. 8, 2013, no. 17, 807 - 828 HIKARI Ltd, www.m-hikari.com

\title{
Stochastic Incremental Approach for Modelling the Claims Reserves
}

\author{
Ilyes Chorfi \\ Department of Mathematics \\ University of Badji Mokhtar-Annaba, Annaba, Algeria \\ ch.ilyes@hotmail.fr \\ M. Riad Remita \\ Department of Mathematics \\ University of Badji Mokhtar-Annaba, Annaba, Algeria \\ riad.remita@univ-annaba.org
}

Copyright (c) 2013 Ilyes Chorfi and M. Riad Remita. This is an open access article distributed under the Creative Commons Attribution License, which permits unrestricted use, distribution, and reproduction in any medium, provided the original work is properly cited.

\begin{abstract}
The paper focuses on calculation of claims reserves using a stochastic incremental approach (stochastic Chain Ladder using only incremental payments), and for that we establish the require formulae and we put our assumptions by modifying Mack's model. We will next concentrate on the calendar year view of the development triangle, to clarify more we propose a new tabulation form, then we apply the CDR for both cases (incremental approach and calendar year view). By using the incremental vision we avoid a step of calculation (no need to calculate the cumulative triangle), and we got identical results with easier formulae which brings lot of advantages for insurance companies.
\end{abstract}

Mathematics Subject Classification: 62P05, 97M30

Keywords: Stochastic Claims Reserving, Chain-Ladder Method, Mack's Model, CDR, Incremental Payments, Calendar year view 


\section{Introduction}

Payment of claims does not always take place at once, in the same accident year. The regulation of claims is done over time, and it is necessary to establish reserves to honor future liabilities. As the claims amount, that will be finally paid are unknown currently. The amount to put in reserve is also unknown and must be estimated. These estimations can be calculate by the so-called technical IBNR (Incurred But Not Reported), which are based on the past claims payments to estimate its future development. In this paper we will study a new approaches, more formally a modern and sophistical vision to old techniques, for estimation of loss reserves. So we will first present the so-called stochastic incremental approach which calculate the reserves and the ultimate claims using only incremental payments of development triangle, and the second approach is a modern presentation of an old vision, the incremental approach in calendar year view $(t)$ which is already mentioned by Buchwalder-BuhlmannMerz-Wuthrich[1], Eisele-Artzner[2], Partrat-Pey-Schilling[7], in our case we study the approach in details, establish the formulae and propose a new tabulation form to give a clear view for the calendar years.

A claims development triangle has three directions:

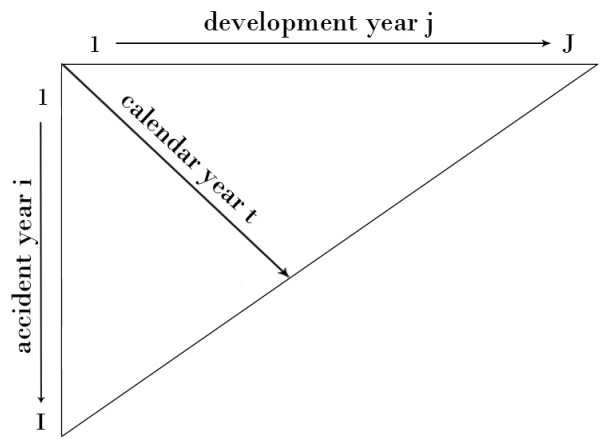

Figure 1: Three directions of claims development triangle

The two directions, development year and accident year, are orthogonal, but the calendar year direction is not orthogonal neither to the accident year direction nor to the development year direction. The paper is organized as follows. In section 2, we will present the stochastic incremental approach which is the incremental case of the Mack's model, so we will reformulate the assumptions and establish the formulae require basing only on incremental payments, which include the error estimation on calculation of reserves, then we will apply the CDR for this approach. Section 3 present the incremental approach in calendar year view, so we propose a new form of tabulation figure 5 to clarify this vision, follow by the apply of the CDR. To illustrate the results, a 
numerical example with conclusion is provided in Section 4, the example show the uses of the two approaches studied, and the results obtained are identical of those using traditional claims reserving techniques for run-off triangles, in addition of these results we can get the reserve of each calendar year $(t)$.

\section{Presentation of the incremental approach}

In this section we will base only on the incremental payments data $D_{i, j}$ to estimate the parameters. This mean we don't need to go a step forward to calculate the cumulated payments as it's done in traditional claims reserving techniques, otherwise said, we want show that we can find the same results of traditional claims reserving techniques based on cumulated development triangle, directly by the incremental development triangle and for that we present the approach as follow. First presentation of uses notations. Secondly we will present the assumptions of the approach by modifying Mack's Model, and show that the development will be conditioned to the incremental payments data, then we will establish the formulae for estimating the parameters. Next we will estimate the error in calculation of reserves. After that we will make comparison between Mack's formulae and the formulae given by the incremental approach, finally we apply the CDR for this approach. To begin with, we define the notations used in this paper, and we denote:

$i \in\{1, \ldots, I\}$ accident years,

$j \in\{1, \ldots, J\}$ development years,

For simplicity, we assume that $I=J$.

$X_{i, j}$ incremental payments with $S_{i, j}=\sum_{l=1}^{j} X_{i, l}$.

Provisioning is a prediction problem, conditioned by the information available at time $\mathrm{t}=\mathrm{I}$.

For that we denote $D_{i, j}$ the set of all data available at time $\mathrm{t}=\mathrm{I}$, more formally $D_{i, j}=\sigma\left\{X_{i, j} \mid i+j \leq I+1\right\}$.

If we focus on the set of all data observed until the development year $j$, we note $D_{j}=\sigma\left\{X_{i, l} \mid i+l \leq I+1, l \leq j\right\}$.

\subsection{Assumptions' modification of Mack's model}

This new approach is also based on three assumptions, the two first assumptions are:

H1 : We have, $\mathbb{E}\left[X_{i, j+1} \mid X_{i, 1}, \ldots, X_{i, j}\right]=S_{i, j} \cdot\left(f_{j}-1\right)$, for all $i \in\{1, \ldots, I\}, j \in\{1, \ldots, J-1\}$, where $f_{j}$ are the development factors. H2 : The variables $\left\{X_{i, 1}, \ldots, X_{i, I}\right\}$ and $\left\{X_{k, 1}, \ldots, X_{k, I}\right\}$ of different accident years $i \neq k$ are independent. 

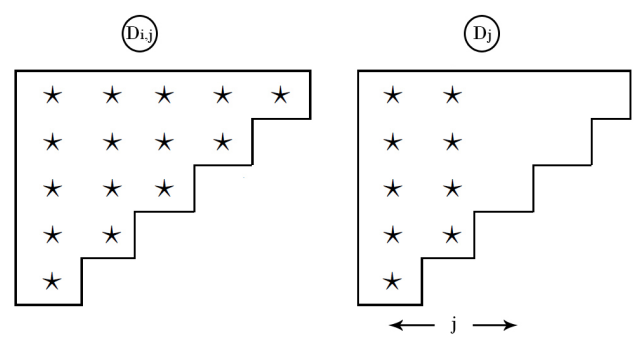

Figure 2: The information available to make the prediction.

\subsection{Estimation of parameters}

\subsubsection{The development factors}

For each development year $j \in\{1, \ldots, J-1\}$, the development factors $f_{j}$ (Chain ladder factors) are estimated by

$$
\widehat{f}_{j}=\frac{\sum_{i=1}^{I-j} X_{i, j+1}}{\sum_{i=1}^{I-j} S_{i, j}}+1 .
$$

We can not guess the true values of the development factors $f_{1}, \ldots, f_{J-1}$ from data because the whole run-off triangular is not yet known at time $\mathrm{t}=\mathrm{I}$. They only can be estimated using incremental payments $X_{i, j}$ as we showed in the formula (1), One prominent property of a good estimator is that the estimator should be unbiased.

Theorem 2.1. Under the assumptions $H 1$ and $H 2$, the estimation of the development factors $\widehat{f}_{1}, \ldots, \widehat{f}_{J-1}$ defined by (1) are unbiased and uncorrelated.

Proof $\boldsymbol{A}$. We will first demonstrate the unbiasedness of development factors, i.e. that $\mathbb{E}\left(\widehat{f}_{j}\right)=f_{j}$.

Because of the iterative rule for expectations,

$$
\mathbb{E}\left(\widehat{f}_{j}\right)=\mathbb{E}\left(\mathbb{E}\left[\widehat{f}_{j} \mid D_{j}\right]\right)
$$

We have

$$
\begin{aligned}
\mathbb{E}\left[X_{i, j+1} \mid D_{j}\right] & =\mathbb{E}\left[X_{i, j+1} \mid X_{i, 1}, \ldots, X_{i, j}\right], \text { by } \mathbf{H 2} \\
& =S_{i, j} \cdot\left(f_{j}-1\right), \text { by } \mathbf{H} \mathbf{1}
\end{aligned}
$$


This implies that

$$
\begin{aligned}
\mathbb{E}\left[\widehat{f}_{j} \mid D_{j}\right] & =\mathbb{E}\left[\frac{\sum_{i=1}^{I-j} X_{i, j+1}}{\sum_{i=1}^{I-j} S_{i, j}}+1 \mid D_{j}\right] \\
& =\frac{\sum_{i=1}^{I-j} \mathbb{E}\left[X_{i, j+1} \mid D_{j}\right]}{\sum_{i=1}^{I-j} S_{i, j}}+1, \text { because } S_{i, j} \text { is } D_{j} \text {-measurable } \\
& =\frac{\left(f_{j}-1\right) \cdot \sum_{i=1}^{I-j} S_{i, j}}{\sum_{i=1}^{I-j} S_{i, j}}+1=f_{j} .
\end{aligned}
$$

$\mathbb{E}\left(\widehat{f}_{j}\right)=\mathbb{E}\left(\mathbb{E}\left[\widehat{f}_{j} \mid D_{j}\right]\right)=\mathbb{E}\left(f_{j}\right)=f_{j}$. Which shows that the estimators $\widehat{f}_{j}$ are unbiased.

We turn now to the no correlation between the estimators of the development factors, i.e. that $\mathbb{E}\left(\widehat{f}_{j_{1}} \cdot \widehat{f}_{j_{2}}\right)=\mathbb{E}\left(\widehat{f}_{j_{1}}\right) \cdot \mathbb{E}\left(\widehat{f}_{j_{2}}\right)$ for $j_{1}\left\langle j_{2}\right.$,

$$
\begin{aligned}
\mathbb{E}\left(\widehat{f}_{j_{1}} \cdot \widehat{f}_{j_{2}}\right) & =\mathbb{E}\left(\mathbb{E}\left[\widehat{f}_{j_{1}} \cdot \widehat{f}_{j_{2}} \mid D_{j_{2}}\right]\right) \\
& =\mathbb{E}\left(\widehat{f}_{j_{1}} \cdot \mathbb{E}\left[\widehat{f}_{j_{2}} \mid D_{j_{2}}\right]\right), \text { because } j_{1}<j_{2} \\
& =\mathbb{E}\left(\widehat{f}_{j_{1}}\right) \cdot f_{j_{2}} \\
& =\mathbb{E}\left(\widehat{f}_{j_{1}}\right) \cdot \mathbb{E}\left(\widehat{f}_{j_{2}}\right), \text { because } \widehat{f}_{j} \text { are unbiased. }
\end{aligned}
$$

\subsubsection{The ultimate claims amount}

The aim of the chain ladder method and every claims reserving method is the estimation of the ultimate claims amount $\widetilde{Z}_{i}$ for the accident years $i=2, \ldots, I$. In our approach we can calculate $\widehat{\widetilde{Z}}_{i}$ using two formulae, the first one is so easy, by using the summation of all incremental payments.

$$
\begin{aligned}
\widehat{\widetilde{Z}}_{i} & =\sum_{j=1}^{I-i+1} X_{i, j}+\sum_{j=I-i+2}^{J} \widehat{X}_{i, j} \\
& =Z_{i}^{0}+\widehat{R}_{i} .
\end{aligned}
$$

Where the first term represents a known part (the yearly past obligations) $Z_{i}^{0}$, and the second term corresponds the outstanding claims reserves $\widehat{R}_{i}$, or we can simply using the classic form given by

$$
\widehat{\widetilde{Z}}_{i}=Z_{i}^{0} \cdot\left(\widehat{f}_{I-i+1} \ldots \widehat{f}_{J-1}\right) .
$$

Theorem 2.2. Under the assumptions $H 1$ and $H 2$, the estimation of the ultimate claims amount $\widehat{\widetilde{Z}}_{i}$ defined by (3) are unbiased. 


\section{Proof $B$.}

We have

$$
\begin{aligned}
\mathbb{E}\left(\widehat{\widetilde{Z}}_{i}\right)= & \mathbb{E}\left(Z_{i}^{0} \cdot \widehat{f}_{I-i+1} \ldots \widehat{f}_{J-1}\right) \\
= & \mathbb{E}\left(\mathbb{E}\left[Z_{i}^{0} \cdot \widehat{f}_{I-i+1} \ldots \widehat{f}_{J-1} \mid D_{J-1}\right]\right) \\
= & \mathbb{E}\left(Z_{i}^{0} \cdot \mathbb{E}\left[\widehat{f}_{I-i+1} \ldots \widehat{f}_{J-1} \mid D_{J-1}\right]\right), \\
& \text { because } Z_{i}^{0} \text { is } D_{J-1} \text {-measurable } \\
= & \mathbb{E}\left(Z_{i}^{0} \cdot \widehat{f}_{I-i+1} \ldots \widehat{f}_{J-2} \cdot \mathbb{E}\left[\widehat{f}_{J-1} \mid D_{J-1}\right]\right), \\
& \text { also } \widehat{f}_{I-i+1}, \ldots, \widehat{f}_{J-2} \text { is } D_{J-1} \text {-measurable } \\
= & \mathbb{E}\left(Z_{i}^{0} \cdot \widehat{f}_{I-i+1} \ldots \widehat{f}_{J-2}\right) \cdot f_{J-1} \\
\text { then } & \\
\mathbb{E}\left(\widehat{\widetilde{Z}}_{i}\right)= & Z_{i}^{0} \cdot f_{I-i+1} \ldots f_{J-2} \cdot f_{J-1}, \\
& \text { by repeated the operation } \\
= & \widetilde{Z}_{i} .
\end{aligned}
$$

\subsubsection{Yearly claims reserves}

One of the advantages of our new approach is that we can easily calculate the outstanding claims reserves estimators (in other words, what is left to pay for claims incurred in year i), by a simple sum of the incremental payments estimators for $i \in\{2, \ldots, I\}$

$$
\widehat{R}_{i}=\sum_{j=I-i+2}^{J} \widehat{X}_{i, j}
$$

We can also obtain $\widehat{R}_{i}$ using the classic principle, i.e.(make the difference between the estimators of the ultimate claims amount $\widehat{\widetilde{Z}}_{i}$ and the past obligations $Z_{i}^{0}$ has already been paid up to now), and we denote for $i \in\{2, \ldots, I\}$

$$
\widehat{R}_{i}=\widehat{\widetilde{Z}}_{i}-Z_{i}^{0}=Z_{i}^{0} \cdot\left[\prod_{j=I-i+1}^{J-1} \widehat{f}_{j}-1\right]
$$


and their sum will give

$$
\begin{aligned}
\widehat{R}=\sum_{i=2}^{I} \widehat{R}_{i} & =\sum_{i=2}^{I}\left[\widehat{\widetilde{Z}}_{i}-Z_{i}^{0}\right] \\
& =\sum_{i=2}^{I} Z_{i}^{0} \cdot\left[\prod_{j=I-i+1}^{J-1} \widehat{f}_{j}-1\right] .
\end{aligned}
$$

we observe here that we can also get the overall reserve by a simple summation of all the future incremental payments in the lower part of development triangle,

$$
\widehat{R}=\sum_{i=2}^{I} \sum_{j=I-i+2}^{J} \widehat{X}_{i, j} .
$$

Theorem 2.3. Under the assumptions $H 1$ and $H 2$, the estimation of the outstanding claims reserves $\widehat{R}_{i}$ and the estimation overall reserve $\widehat{R}$ are unbiased.

Proof $C$.

$$
\begin{aligned}
\mathbb{E}\left(\widehat{R}_{i}\right) & =\mathbb{E}\left(\widehat{\widetilde{Z}}_{i}-Z_{i}^{0}\right) \\
& =\mathbb{E}\left(\widehat{\widetilde{Z}}_{i}\right)-\mathbb{E}\left(Z_{i}^{0}\right) \\
& =\widetilde{Z}_{i}-Z_{i}^{0}=R_{i} .
\end{aligned}
$$

and

$$
\mathbb{E}(\widehat{R})=\mathbb{E}\left(\sum_{i=2}^{I} \widehat{R}_{i}\right)=\sum_{i=2}^{I} R_{i}=R
$$

\subsection{Calculation of standard error}

The formula for estimating the standard error is based on Mack's model, and in this paper we will measure this uncertainty using only the incremental payments.

\subsubsection{The estimation error of the yearly claims reserves}

$\widehat{\widetilde{Z}}_{i}$ provides an estimator but not the exact value of $\widetilde{Z}_{i}$. In this section we are interested in the average distance between the estimator and the true value. The mean square error $M S E\left(\widehat{\widetilde{Z}}_{i}\right)$ is defined by

$$
\operatorname{MSE}\left(\widehat{\widetilde{Z}}_{i}\right)=\mathbb{E}\left[\left(\widehat{\widetilde{Z}}_{i}-\widetilde{Z}_{i}\right)^{2} \mid D_{i, j}\right]
$$


Here we are interested in a conditional mean based on the specific incremental payments data set $\left(D_{i, j}\right)$ to obtain the mean deviation between $\widehat{\widetilde{Z}}_{i}$ and $\widetilde{Z}_{i}$ only due to future randomness.

The standard error $S E\left(\widehat{\widetilde{Z}}_{i}\right)$ is defined as equal to $\sqrt{M S E\left(\widehat{\widetilde{Z}}_{i}\right)}$. If we are interested in the error on the provision, we must calculate

$$
\operatorname{MSE}\left(\widehat{R}_{i}\right)=\mathbb{E}\left[\left(\widehat{R}_{i}-R_{i}\right)^{2} \mid D_{i, j}\right]
$$

such as $\widehat{R}_{i}=\widehat{\widetilde{Z}}_{i}-Z_{i}^{0}$ and $R_{i}=\widetilde{Z}_{i}-Z_{i}^{0}$ we finally obtain

$$
\begin{aligned}
\operatorname{MSE}\left(\widehat{R}_{i}\right) & =\mathbb{E}\left[\left(\widehat{R}_{i}-R_{i}\right)^{2} \mid D_{i, j}\right] \\
& =\mathbb{E}\left[\left(\widehat{\widetilde{Z}}_{i}-\widetilde{Z}_{i}\right)^{2} \mid D_{i, j}\right]=\operatorname{MSE}\left(\widehat{\widetilde{Z}}_{i}\right) .
\end{aligned}
$$

The error in estimating the yearly claims reserves is equal to the estimation error of the ultimate claims amount.

In order to calculate $M S E\left(\widehat{\widetilde{Z}}_{i}\right)$ we'll decompose it according to the following formula:

$$
\operatorname{MSE}\left(\widehat{R}_{i}\right)=\operatorname{Var}\left(\widetilde{Z}_{i} \mid D_{i, j}\right)+\left(\mathbb{E}\left[\widetilde{Z}_{i} \mid D_{i, j}\right]-\widehat{\widetilde{Z}}_{i}\right)^{2}
$$

This formula shows that we therefore need to estimate the variance of $X_{i, j}$, this will lead us to identify a third assumption for our new approach derived from Mack's Model,

H3 $: \operatorname{Var}\left(X_{i, j+1} \mid X_{i, 1}, \ldots, X_{i, j}\right)=S_{i, j} \cdot \sigma_{j}^{2}, \quad$ for $1 \leq i \leq I, 1 \leq j \leq J-1$.

The parameters $\sigma_{j}^{2}$ are unknown and must be estimated.

Property 2.4. Under $H 1, H 2$ and $H 3$,

$$
\widehat{\sigma}_{j}^{2}=\frac{1}{I-j-1} \sum_{i=1}^{I-j} S_{i, j} \cdot\left(\frac{X_{i, j+1}}{S_{i, j}}+1-\widehat{f}_{j}\right)^{2}, \text { for } 1 \leq j \leq J-2
$$

is an unbiased estimator of $\sigma_{j}^{2}$ and

$$
\widehat{\sigma}_{J-1}^{2}=\min \left(\frac{\widehat{\sigma}_{J-2}^{4}}{\widehat{\sigma}_{J-3}^{2}}, \min \left(\widehat{\sigma}_{J-3}^{2}, \widehat{\sigma}_{J-2}^{2}\right)\right) \text {, see Mack [4]. }
$$


Proof D. The formula (D1) Comes from

$$
\begin{aligned}
\sigma_{j}^{2} & =\frac{\operatorname{Var}\left(X_{i, j+1} \mid X_{i, 1}, \ldots, X_{i, j}\right)}{S_{i, j}} \\
& =\operatorname{Var}\left(\frac{X_{i, j+1}}{\sqrt{S_{i, j}}} \mid X_{i, 1}, \ldots, X_{i, j}\right) \\
& =\frac{1}{I-j-1} \sum_{i=1}^{I-j}\left(\frac{X_{i, j+1}}{\sqrt{S_{i, j}}}-\mathbb{E}\left(\frac{X_{i, j+1}}{\sqrt{S_{i, j}}} \mid X_{i, 1}, \ldots, X_{i, j}\right)\right)^{2} \\
& =\frac{1}{I-j-1} \sum_{i=1}^{I-j}\left(\frac{X_{i, j+1}}{\sqrt{S_{i, j}}}-\frac{\mathbb{E}\left(X_{i, j+1} \mid X_{i, 1}, \ldots, X_{i, j}\right)}{\sqrt{S_{i, j}}}\right)^{2} \\
& =\frac{1}{I-j-1} \sum_{i=1}^{I-j} S_{i, j}\left(\frac{X_{i, j+1}}{S_{i, j}}+1-f_{j}\right)^{2} \text { by } H 1 .
\end{aligned}
$$

We get $\widehat{\sigma}_{j}^{2}$ by replacing the unknown parameters $f_{j}$, with their unbiased estimators $\widehat{f}_{j}$.

$$
\widehat{\sigma}_{j}^{2}=\frac{1}{I-j-1} \sum_{i=1}^{I-j} S_{i, j} \cdot\left(\frac{X_{i, j+1}}{S_{i, j}}+1-\widehat{f}_{j}\right)^{2} .
$$

Theorem 2.5. Under the assumptions $H 1, H 2$ and $H 3, \widehat{\sigma}_{j}^{2}$ are unbiased.

Proof $\boldsymbol{E}$. The definition of $\widehat{\sigma}_{j}^{2}$ can be rewritten as

$$
\begin{aligned}
(I-j-1) \cdot \widehat{\sigma}_{j}^{2} & =\sum_{i=1}^{I-j} S_{i, j} \cdot\left(\frac{X_{i, j+1}+S_{i, j}}{S_{i, j}}-\widehat{f}_{j}\right)^{2} \\
& =\sum_{i=1}^{I-j}\left(\frac{\left(X_{i, j+1}+S_{i, j}\right)^{2}}{S_{i, j}}-2 \cdot\left(X_{i, j+1}+S_{i, j}\right) \times \widehat{f}_{j}+S_{i, j} \cdot \widehat{f}_{j}^{2}\right. \\
& =\sum_{i=1}^{I-j} \frac{\left(X_{i, j+1}+S_{i, j}\right)^{2}}{S_{i, j}}-\sum_{i=1}^{I-j} S_{i, j} \cdot \widehat{f}_{j}^{2},
\end{aligned}
$$

then

$$
\begin{aligned}
\mathbb{E}\left((I-j-1) \cdot \widehat{\sigma}_{j}^{2} \mid D_{j}\right)= & \sum_{i=1}^{I-j} \underbrace{\mathbb{E}\left(\left(X_{i, j+1}+S_{i, j}\right)^{2} \mid D_{j}\right)}_{(E 3)} / S_{i, j} \\
& -\sum_{i=1}^{I-j} S_{i, j} \cdot \underbrace{\mathbb{E}\left(\widehat{f}_{j}^{2} \mid D_{j}\right)}_{(E 4)},
\end{aligned}
$$


because $S_{i, j}$ is $D_{j}$-measurable, we calculate first (E3) by

$$
\begin{aligned}
\mathbb{E}\left[\left(X_{i, j+1}+S_{i, j}\right)^{2} \mid D_{j}\right]= & \mathbb{E}\left[\left(X_{i, j+1}+S_{i, j}\right)^{2} \mid X_{i, 1}, \ldots, X_{i, j}\right], \text { by } H 2 \\
= & \operatorname{Var}\left[\left(X_{i, j+1}+S_{i, j}\right) \mid X_{i, 1}, \ldots, X_{i, j}\right] \\
& +\mathbb{E}\left[\left(X_{i, j+1}+S_{i, j}\right) \mid X_{i, 1}, \ldots, X_{i, j}\right]^{2} \\
= & \operatorname{Var}\left(X_{i, j+1} \mid X_{i, 1}, \ldots, X_{i, j}\right) \\
& +\left[\mathbb{E}\left(X_{i, j+1} \mid X_{i, 1}, \ldots, X_{i, j}\right)+S_{i, j}\right]^{2} \\
= & S_{i, j} \cdot \sigma_{j}^{2}+\left[S_{i, j} \cdot f_{j}\right]^{2}, \text { by } H 1 \text { and } H 3 .
\end{aligned}
$$

We turn now to $(E 4)$ for that we using $(F 6)$ and $(A 3)$ we obtain

$$
\mathbb{E}\left(\widehat{f}_{j}^{2} \mid D_{j}\right)=\operatorname{Var}\left(\widehat{f}_{j} \mid D_{j}\right)+\left[\mathbb{E}\left(\widehat{f}_{j} \mid D_{j}\right)\right]^{2}=\frac{\sigma_{j}^{2}}{\sum_{i=1}^{I-j} S_{i, j}}+f_{j}^{2}
$$

Inserting $(E 3)$ and $(E 4)$ into $(E 2)$ we obtain

$$
\begin{aligned}
\mathbb{E}\left((I-j-1) \cdot \widehat{\sigma}_{j}^{2} \mid D_{j}\right) & =\sum_{i=1}^{I-j}\left(\sigma_{j}^{2}+S_{i, j} \cdot f_{j}^{2}\right)-\sum_{i=1}^{I-j} \frac{S_{i, j} \cdot \sigma_{j}^{2}}{\sum_{i=1}^{I-j} S_{i, j}}+S_{i, j} \cdot f_{j}^{2} \\
& =(I-j-1) \cdot \sigma_{j}^{2}
\end{aligned}
$$

The following theorem gives the formula for estimating $\operatorname{MSE}\left(\widehat{R}_{i}\right)$.

Theorem 2.6. Under the assumptions $H 1, H 2$ and $H 3, M S E\left(\widehat{R}_{i}\right)$ can be estimated by

$$
\widehat{M S E}\left(\widehat{R}_{i}\right)=\widehat{\widetilde{Z}}_{i}^{2} \cdot \sum_{j=I-i+1}^{J-1} \frac{\widehat{\sigma}_{j}^{2}}{\widehat{f}_{j}^{2}}\left(\frac{1}{\widehat{S}_{i, j}}+\frac{1}{\sum_{l=1}^{I-j} S_{l, j}}\right)
$$

Proof $\boldsymbol{F}$. We use the abbreviations

$$
\begin{aligned}
\mathbb{E}_{i}(Y) & =\mathbb{E}\left(Y \mid X_{i, 1}, \ldots, X_{i, I-i+1}\right), \\
\operatorname{Var}_{i}(Y) & =\operatorname{Var}\left(Y \mid X_{i, 1}, \ldots, X_{i, I-i+1}\right) .
\end{aligned}
$$

The theorem of conditional variance

$$
\operatorname{Var}(Y)=\mathbb{E}[\operatorname{Var}(Y \mid X)]+\operatorname{Var}[\mathbb{E}(Y \mid X)],
$$

we have the formula (8) given by

$$
\operatorname{MSE}\left(\widehat{R}_{i}\right)=\underbrace{\operatorname{Var}\left(\widetilde{Z}_{i} \mid D_{i, j}\right)}_{\text {Error Process }}+\underbrace{\left(\mathbb{E}\left(\widetilde{Z}_{i} \mid D_{i, j}\right)-\widehat{\widetilde{Z}}_{i}\right)^{2}}_{\text {Error Estimators }} .
$$


Using assumption $H 2$ and then repeatedly applying the formula of the assumptions $H 1$ and $H 3$, the first term of $M S E\left(\widehat{R}_{i}\right)$ can be written as:

$$
\begin{aligned}
\operatorname{Var}\left(\widetilde{Z}_{i} \mid D_{i, j}\right)= & \operatorname{Var}_{i}\left(\widetilde{Z}_{i}\right), H 2 \\
= & \left.\mathbb{E}_{i}\left[\operatorname{Var}_{\bar{Z}} \mid X_{i, 1}, \ldots, X_{i, J-1}\right)\right]+\operatorname{Var}_{i}\left[\mathbb{E}\left(\widetilde{Z}_{i} \mid X_{i, 1}, \ldots, X_{i, J-1}\right)\right] \\
= & \mathbb{E}_{i}\left(S_{i, J-1} \cdot \sigma_{J-1}^{2}\right)+\operatorname{Var}_{i}\left(S_{i, J-1}+S_{i, J-1} \cdot\left(f_{J-1}-1\right)\right), \text { by } H 3, H 1 \\
= & \mathbb{E}_{i}\left(S_{i, J-1}\right) \cdot \sigma_{J-1}^{2}+\operatorname{Var}_{i}\left(S_{i, J-1}\right) \cdot f_{J-1}^{2} \\
= & \mathbb{E}_{i}\left[S_{i, J-2} \cdot\left(f_{J-2}-1\right)+S_{i, J-2}\right] \cdot \sigma_{J-1}^{2}+f_{J-1}^{2} \cdot \mathbb{E}_{i}\left(S_{i, J-2} \cdot \sigma_{J-2}^{2}\right) \\
& +f_{J-1}^{2} \cdot \operatorname{Var}_{i}\left[S_{i, J-2}+S_{i, J-2} \cdot\left(f_{J-2}-1\right)\right] \\
= & \mathbb{E}_{i}\left(S_{i, J-2}\right) \cdot f_{J-2} \cdot \sigma_{J-1}^{2}+\mathbb{E}_{i}\left(S_{i, J-2}\right) \cdot f_{J-1}^{2} \cdot \sigma_{J-2}^{2} \\
& +\operatorname{Var}_{i}\left[S_{i, J-2}\right] \cdot f_{J-1}^{2} \cdot f_{J-2}^{2} \\
\quad & \quad \vdots \\
= & Z_{i}^{0} \cdot \sum_{j=I+1-i}^{J-1}\left(f_{I+1-i} \ldots f_{j-1}\right) \times \sigma_{j}^{2} \cdot\left(f_{j+1}^{2} \ldots f_{J-1}^{2}\right) .
\end{aligned}
$$

As we don't know the parameters $f_{j}$ et $\sigma_{j}^{2}$, we replace them by their estimators $\widehat{f}_{j}$ et $\widehat{\sigma}_{j}^{2}$, that is to say that we estimate the first term of the expression $(F 4)$ for $M S E\left(\widehat{R}_{i}\right)$ by

$$
\begin{aligned}
\operatorname{Var}\left(\widetilde{Z}_{i} \mid D_{i, j}\right) & =Z_{i}^{0} \cdot \sum_{j=I-i+1}^{J-1} \widehat{f}_{I-i+1} \ldots \widehat{f}_{j-1} \cdot \widehat{\sigma}_{j}^{2} \cdot \widehat{f}_{j+1}^{2} \ldots \widehat{f}_{J-1}^{2} \\
& =Z_{i}^{0} \cdot \sum_{j=I-i+1}^{J-1} \frac{\widehat{f}_{I-i+1}^{2} \ldots \widehat{f}_{j-1}^{2} \cdot \widehat{f}_{j}^{2} \cdot \widehat{\sigma}_{j}^{2} \cdot \widehat{f}_{j+1}^{2} \ldots \widehat{f}_{J-1}^{2}}{\widehat{f}_{I-i+1} \ldots \widehat{f}_{j-1} \cdot \widehat{f}_{j}^{2}} \\
& =\left(Z_{i}^{0}\right)^{2} \cdot \sum_{j=I+1-i}^{J-1} \frac{\widehat{f}_{I-i+1}^{2} \ldots \widehat{f}_{J-1}^{2} \cdot \widehat{\sigma}_{j}^{2}}{Z_{i}^{0} \cdot \widehat{f}_{I-i+1} \ldots \widehat{f}_{j-1} \cdot \widehat{f}_{j}^{2}} \\
& =\left(Z_{i}^{0}\right)^{2} \cdot \widehat{f}_{I-i+1}^{2} \ldots \widehat{f}_{J-1}^{2} \cdot \sum_{j=I-i+1}^{J-1} \frac{\widehat{\sigma}_{j}^{2} / \widehat{f}_{j}^{2}}{Z_{i}^{0} \cdot \widehat{f}_{I-i+1} \ldots \widehat{f}_{j-1}}
\end{aligned}
$$

So

$$
\left.\operatorname{Var} \widehat{\left(\widetilde{Z}_{i} \mid\right.} D_{i, j}\right)=\left(\widehat{\widetilde{Z}}_{i}\right)^{2} \cdot \sum_{j=I+1-i}^{J-1} \frac{\widehat{\sigma}_{j}^{2} / \widehat{f}_{j}^{2}}{\widehat{S}_{i, j}} .
$$

We turn now to the second term of the expression $(F 4)$ for $M S E\left(\widehat{R}_{i}\right)$

$$
\left(\mathbb{E}\left(\widetilde{Z}_{i} \mid D_{i, j}\right)-\widehat{\widetilde{Z}}_{i}\right)^{2}=\left(Z_{i}^{0}\right)^{2} \cdot\left(f_{I-i+1} \ldots f_{J-1}-\widehat{f}_{I-i+1} \ldots \widehat{f}_{J-1}\right)^{2}
$$

To estimate the second term we cannot simply replace $f_{j}$ by their estimator because this will lead to cancel it. We therefore use another approach. We assume that,

$$
\begin{array}{r}
F=f_{I-i+1} \ldots f_{J-1}-\widehat{f}_{I-i+1} \ldots \widehat{f}_{J-1}=T_{I-i+1}+\ldots+T_{J-1}, \\
\text { where } T_{j}=\widehat{f}_{I-i+1} \ldots \widehat{f}_{j-1} \cdot\left(f_{j}-\widehat{f}_{j}\right) \cdot f_{j+1} \ldots f_{J-1},
\end{array}
$$


so we have

$$
F^{2}=\left(T_{I+1-i}+\ldots+T_{J-1}\right)^{2}=\sum_{j=I-i+1}^{J-1} T_{j}^{2}+2 \sum_{l .\langle j} T_{l} T_{j}
$$

Then we approximate $T_{j}^{2}$ by $\mathbb{E}\left(T_{j}^{2} \mid D_{j}\right)$ et $T_{l}, T_{j}$ par $\mathbb{E}\left(T_{l} T_{j} \mid D_{j}\right)$. As, $\mathbb{E}\left(f_{j}-\widehat{f}_{j} \mid D_{j}\right)=0$ (because $\widehat{f}_{j}$ is an unbiased), we have

$$
\mathbb{E}\left(T_{l} T_{j} \mid D_{j}\right)=0, \text { for } l<j,
$$

so

$$
\begin{gathered}
F^{2}=\sum_{j=I+1-i}^{J-1} \mathbb{E}\left(T_{j}^{2} \mid D_{j}\right)+2 \sum_{i\langle j} \mathbb{E}\left(T_{i} T_{j} \mid D_{j}\right)=\sum_{j=I+1-i}^{J-1} \mathbb{E}\left(T_{j}^{2} \mid D_{j}\right) . \\
\text { Or } \mathbb{E}\left(T_{j}^{2} \mid D_{j}\right)=\widehat{f}_{I+1-i}^{2} \ldots \widehat{f}_{j-1}^{2} \cdot \mathbb{E}\left(\left(f_{j}-\widehat{f}_{j}\right)^{2} \mid D_{j}\right) \cdot f_{j+1}^{2} \ldots f_{J-1}^{2} .
\end{gathered}
$$

So we should calculate $\mathbb{E}\left(\left(f_{j}-\widehat{f}_{j}\right)^{2} \mid D_{j}\right)$,

$$
\begin{aligned}
\mathbb{E}\left(\left(f_{j}-\widehat{f}_{j}\right)^{2} \mid D_{j}\right) & =\operatorname{Var}\left(\widehat{f}_{j} \mid D_{j}\right) \\
& =\operatorname{Var}\left(\frac{\sum_{i=1}^{I-j} X_{i, j+1}}{\sum_{i=1}^{I-j} S_{i, j}}+1 \mid D_{j}\right) \\
& =\frac{\sum_{i=1}^{I-j} \operatorname{Var}\left(X_{i, j+1} \mid D_{j}\right)}{\left(\sum_{i=1}^{I-j} S_{i, j}\right)^{2}}=\frac{\sigma_{j}^{2}}{\sum_{i=1}^{I-j} S_{i, j}} .
\end{aligned}
$$

$$
\text { So } \mathbb{E}\left(\left(f_{j}-\widehat{f}_{j}\right)^{2} \mid D_{j}\right)=\operatorname{Var}\left(\widehat{f}_{j} \mid D_{j}\right)=\frac{\sigma_{j}^{2}}{\sum_{i=1}^{I-j} S_{i, j}} \text {. }
$$

We obtain

$$
\mathbb{E}\left(T_{j}^{2} \mid D_{j}\right)=\frac{\widehat{f}_{I+1-i}^{2} \cdots \widehat{f}_{j-1}^{2} \cdot \sigma_{j}^{2} \cdot f_{j+1}^{2} \ldots f_{J-1}^{2}}{\sum_{i=1}^{I-j} S_{i, j}}
$$

so we estimate $F^{2}$ by $\sum_{j=I-i+1}^{J-1} \mathbb{E}\left(T_{j}^{2} \mid D_{j}\right)$ and we can now replace the parameters $f_{j}$ et $\sigma_{j}^{2}$ by their unbiased estimators $\widehat{f}_{j}$ et $\widehat{\sigma}_{j}^{2}$, that is to say that we estimate the second term of $M S E\left(\widehat{R}_{i}\right)$, 


$$
\begin{aligned}
\left(\mathbb{E}\left(\widetilde{Z}_{i} \mid \widehat{D_{i, j}}\right)-\widehat{\widetilde{Z}}_{i}\right)^{2} & =\left(\widehat{\widetilde{Z}}_{i}\right)^{2} \cdot \sum_{j=I-i+1}^{J-1} \frac{\widehat{f}_{I+1-i}^{2} \cdots \widehat{f}_{j-1}^{2} \cdot \widehat{\sigma}_{j}^{2} \cdot \widehat{f}_{j+1}^{2} \cdots \widehat{f}_{J-1}^{2}}{\sum_{i=1}^{I-j} S_{i, j}} \\
& =\left(\widehat{\widetilde{Z}}_{i}\right)^{2} \cdot \sum_{j=I-i+1}^{J-1} \frac{\widehat{\sigma}_{j}^{2} / \widehat{f}_{j}^{2}}{I-j} \sum_{i=1} S_{i, j}
\end{aligned}
$$

By adding the expressions $(F 5)$ and (F7), we find the formula proposed for $\widehat{M S E\left(\widehat{R}_{i}\right)}$

$$
\begin{aligned}
\widehat{M S E\left(\widehat{R}_{i}\right)} & \left.=\operatorname{Var} \widehat{\left(\widetilde{Z}_{i} \mid\right.} D_{i, j}\right)+\left(\mathbb{E}\left(\widetilde{Z}_{i} \mid \widehat{D_{i, j}}\right)-\widehat{\widetilde{Z}}_{i}\right)^{2} \\
& =\left(\widehat{\widetilde{Z}}_{i}\right)^{2} \cdot \sum_{j=I-i+1}^{J-1} \frac{\widehat{\sigma}_{j}^{2} / \widehat{f}_{j}^{2}}{\widehat{S}_{i, j}}+\left(\widehat{\widetilde{Z}}_{i}\right)^{2} \cdot \sum_{j=I-i+1}^{J-1} \frac{\widehat{\sigma}_{j}^{2} / \widehat{f}_{j}^{2}}{I-j} S_{i=1} \\
& =\left(\widehat{\widetilde{Z}}_{i, j}\right)^{2} \cdot \sum_{j=I-i+1}^{J-1} \frac{\widehat{\sigma}_{j}^{2}}{\widehat{f}_{j}^{2}}\left(\frac{1}{\widehat{S}_{i, j}}+\frac{1}{\sum_{i=1}^{I-j} S_{i, j}}\right) .
\end{aligned}
$$

\subsubsection{The estimation error of the overall reserves}

It is also interesting to calculate the error on the estimated overall reserve $\widehat{R}=\widehat{R}_{2}+\ldots+\widehat{R}_{I}$. We can not simply sum the errors $M S E(\widehat{R})$ because they are correlated by the same estimators $\widehat{f}_{j}$ and $\widehat{\sigma}_{j}^{2}$, but we can use the following theorem.

Theorem 2.7. Under the assumptions $H 1, H 2$ and $H 3, M S E(\widehat{R})$ can be estimated by

$$
\widehat{M S E}(\widehat{R})=\sum_{i=2}^{I}\left[M S E\left(\widehat{R}_{i}\right)+\widehat{\widetilde{Z}}_{i}\left(\sum_{k=i+1}^{I} \widehat{\widetilde{Z}}_{k}\right) \sum_{j=I-i+1}^{J-1} \frac{2 \widehat{\sigma}_{j}^{2} / \widehat{f}_{j}^{2}}{\sum_{k=1}^{I-j} S_{k, j}}\right]
$$

Proof $\boldsymbol{G}$. This proof is analogous to that in (Proof F). The explanations 
will therefore be brief.

$$
\begin{aligned}
\operatorname{MSE}\left(\sum_{i=2}^{I} \widehat{R}_{i}\right) & =\mathbb{E}\left[\left(\sum_{i=2}^{I} \widehat{R}_{i}-\sum_{i=2}^{I} R_{i}\right)^{2} \mid D_{i, j}\right] \\
& =\mathbb{E}\left[\left(\sum_{i=2}^{I} \widehat{\widetilde{Z}}_{i}-\sum_{i=2}^{I} \widetilde{Z}_{i}\right)^{2} \mid D_{i, j}\right] \\
& =\operatorname{Var}\left(\sum_{i=2}^{I} \widetilde{Z}_{i} \mid D_{i, j}\right)+\left(\mathbb{E}\left(\sum_{i=2}^{I} \widetilde{Z}_{i} \mid D_{i, j}\right)-\sum_{i=2}^{I} \widehat{\widetilde{Z}}_{i}\right)^{2} .
\end{aligned}
$$

We have been calculated this terms on (Proof F),

$$
M S E\left(\sum_{i=2}^{I} \widehat{R}_{i}\right)=\sum_{i=2}^{I} M S E\left(\widehat{R}_{i}\right)+\sum_{2 \leqslant i<k \leqslant I} 2 \cdot Z_{i}^{0} Z_{k}^{0} \cdot F_{i} F_{k} .
$$

We therefore need only develop an estimator for $F_{i} F_{k}$. We immediately get the final result of $\widehat{M S E}(\widehat{R})$

$$
\widehat{M S E}(\widehat{R})=\sum_{i=2}^{I}\left[M S E\left(\widehat{R}_{i}\right)+\widehat{\widetilde{Z}}_{i}\left(\sum_{k=i+1}^{I} \widehat{\widetilde{Z}}_{k}\right) \sum_{j=I+1-i}^{J-1} \frac{2 \widehat{\sigma}_{j}^{2} / \widehat{f}_{j}^{2}}{\sum_{k=1}^{I-j} S_{k, j}}\right] .
$$

\subsection{Comparison between Mack's model and incremen- tal approach}

We can see that this new approach using only incremental claims which results easier formulae. Proceeding from the formulae of our approach we can automatically find the same formulae of Mack's model.

\begin{tabular}{|l|l|}
\hline \multicolumn{2}{|c|}{ Mack's Model $\rightleftharpoons$ Incremental Approach } \\
\hline \multicolumn{1}{|c|}{ Mack's Model } & \multicolumn{1}{|c|}{ Incremental Approach } \\
\hline$\widehat{f}_{j}=\frac{\sum_{i=1}^{I-j} C_{i, j+1}}{\sum_{i=1}^{I-j} C_{i, j}}$ & $\widehat{f}_{j}=\frac{\sum_{i=1}^{I-j} X_{i, j+1}}{\sum_{i=1}^{I-j} S_{j}}+1$ \\
\hline$\widehat{C}_{i, J}=C_{i, I-i+1} \times \prod_{j=I-i+1}^{J-1} \widehat{f}_{j}$ & $\widehat{\widetilde{Z}}_{i}=\sum_{j=1}^{I-i+1} X_{i, j}+\sum_{j=I-i+2}^{J} \widehat{X}_{i, j}$ \\
\hline$\widehat{R}_{i}=\widehat{C}_{i, J}-C_{i, I-i+1}$ & $\widehat{R}_{i}=\sum_{j=I-i+2}^{J} \widehat{X}_{i, j}$ \\
\hline$\widehat{R}=\sum_{i=2}^{I} \widehat{R}_{i}$ & $\widehat{R}=\sum_{i=2}^{I} \widehat{R}_{i}$ \\
\hline
\end{tabular}




\subsection{Claim development result}

The difference between two successive estimates of best estimate on time $I$ and $I+1$ is called the claim development result $(C D R)$. We will calculate the CDR using the incremental claims data, for that we will change the notation of the incremental claims data available at time $t=I$ to

$$
D_{i, j}^{I}=\sigma\left\{X_{i, j} ; \quad i+j \leq I+1\right\}
$$

and for the incremental claims data available one period later, at time $t=I+1$ by

$$
D_{i, j}^{I+1}=\left\{X_{i, j} ; i+j \leq I+2 ; i \leq I\right\}=D_{i, j}^{I} \cup\left\{X_{i, J-i+2} ; i \leq I\right\},
$$

"If we go one step ahead in time, we obtain new incremental observations $\left\{X_{i, I-i+2} ; i \leq I\right\}$ on the new diagonal. More formally, this means that we get an enlargement of the $\sigma$-algebra generated by the observations $D_{i, j}^{I}$ to the $\sigma$-algebra generated by the observations $D_{i, j}^{I+1}$, .i.e. $\sigma\left(D_{i, j}^{I}\right) \rightarrow \sigma\left(D_{i, j}^{I+1}\right)$ " from $[6]$.
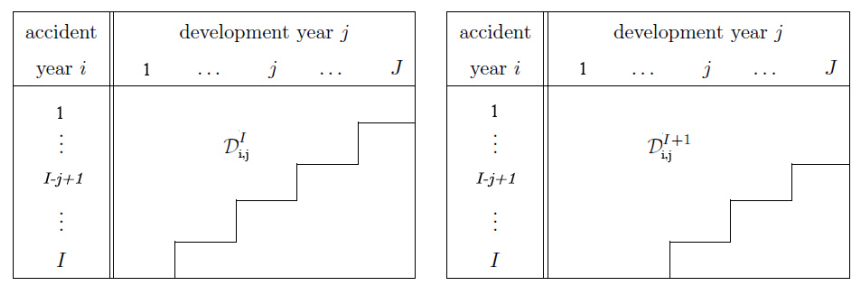

Figure 3: Loss development triangle at time $\mathrm{t}=\mathrm{I}$ and $\mathrm{t}=\mathrm{I}+1$

\subsubsection{Estimators}

The development factors

$$
\widehat{f}_{j}^{I}=\widehat{f}_{j} \quad \text { and } \quad \widehat{f}_{j}^{I+1}=\frac{\sum_{i=1}^{I-j+1} X_{i, j+1}}{\sum_{i=1}^{I-j+1} S_{i, j}}+1 .
$$

The ultimate claims amount

$$
\begin{gathered}
\widehat{\widetilde{Z}}_{i}^{I}=\widehat{\widetilde{Z}}_{i}, \\
\widehat{\widetilde{Z}}_{i}^{I+1}=\sum_{j=1}^{I-i+2} X_{i, j}+\sum_{j=I-i+3}^{J} \widehat{X}_{i, j} \\
=\mathbf{Z}_{i}^{0}+\widehat{R}_{i}^{D_{i, j}^{I+1}} .
\end{gathered}
$$




$$
\text { or } \widehat{\widetilde{Z}}_{i}^{I+1}=\mathbf{Z}_{i}^{0} \cdot \widehat{f}_{I-i+2}^{I+1} \cdots \widehat{f}_{J-1}^{I+1}
$$

Yearly claims reserves

$$
\begin{gathered}
\widehat{R}_{i}^{D_{i, j}^{I}}=\widehat{R}_{i} \\
\widehat{R}_{i}^{D_{i, j}^{I+1}}=\sum_{j=I-i+3}^{J} \widehat{X}_{i, j}, \\
\widehat{R}_{i}^{D_{i, j}^{I+1}}=\widehat{\widetilde{Z}}_{i}^{I+1}-\mathbf{Z}_{i}^{0} .
\end{gathered}
$$

These estimators are unbiased

Definition 2.8 (True CDR for a single accident year). The true CDR for accident year $i \in\{1, \ldots, I\}$ in accounting year $] I, I+1]$ is given by

$$
C D R_{i}(I+1)=\mathbb{E}\left[\widehat{R}_{i}^{I} \mid D_{i, j}^{I}\right]-\left(X_{i, I-i+2}+\mathbb{E}\left[\widehat{R}_{i}^{I+1} \mid D_{i, j}^{I+1}\right]\right)
$$

our approach will permit us to define the $C D R$ as the difference between the matrix of all incremental payments data at time $t=I$ minus the matrix of all incremental payments data at time $t=I+1$.

$$
C D R_{i}(I+1)=\mathbb{E}\left[\widetilde{Z}_{i} \mid D_{i, j}^{I}\right]-\mathbb{E}\left[\widetilde{Z}_{i} \mid D_{i, j}^{I+1}\right] .
$$

Definition 2.9 (Observable CDR, estimator for true CDR). The observable $C D R$ for accident year $i \in\{1, \ldots, I\}$ in accounting year $] I, I+1]$ in the chain ladder method is given by

$$
\widehat{C D R_{i}}(I+1)=\widehat{R}_{i}^{D_{i, j}^{I}}-\left(X_{i, I-i+2}+\widehat{R}_{i}^{D_{i, j}^{I+1}}\right)=\widehat{\widetilde{Z}}_{i}^{I}-\widehat{\widetilde{Z}}_{i}^{I+1} .
$$

$C D R$ real $\left(C D R_{i}(I+1)\right)$ is estimated by the $C D R$ observable $\left.\widehat{C D R}_{i}(I+1)\right)$. See [6]

\subsubsection{The one-year bootstrap}

The stochastic process is applied only to the diagonal of the calendar year $I+1$, this new diagonal has been projected by our approach. Because we use only the incremental claims in this approach, the Bootstrap simulation will be more easy to calculate since the Pearson residuals are based on incremental payments. So we will avoid 3 steps in calculations and we directly calculate the data incremental payments backwards $X_{i, j}^{B}$ by the following formula:

$$
X_{i, j}^{B}=\frac{S_{i, I-i+2}}{\prod_{l=j}^{I-i+1} f_{l}}-\frac{S_{i, I-i+2}}{\prod_{k=j-1}^{I-i+1} f_{k}} .
$$

then we calculate the Pearson residues, and apply the bootstrap n times only for the diagonal we want simulate, using classic formulae see [3]. 


\section{The incremental approach in the calendar years view}

We will focus our interest from the development years $j$ to the calendar years $t$ which give us a new view for the past obligation of each calendar year $t$ and also the estimation of the obligations left to pay of each calendar year $t$ then we can easily calculate the overall reserve by the summation of the past and estimated obligations.

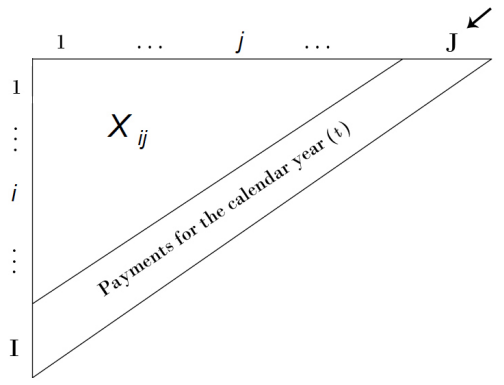

Figure 4: The triangle liquidation in calendar years view

To more clarify this vision we proposed new tabulation form instead of the development triangle.

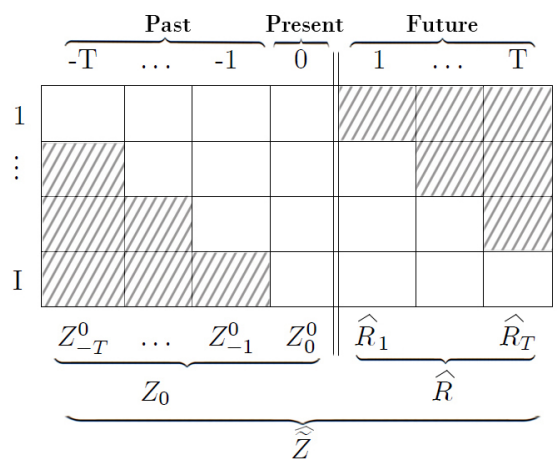

Figure 5: The new form of the triangle liquidation

In this new tabulation, we always represent the accident years by $i \in$ $\{1, \ldots, I\}$ and the calender years by $t \in\{-T, \ldots, T\}$, for $t \in\{-T, \ldots, 0\}$ we have $i \leq t+I$ and for the future part $t \in\{1, \ldots, T\}$ we have $i \geq t+1$. We used negative index $t \in\{-T, \ldots,-1\}$ to represent the past and the current time by $t=0$ and finally the future time by the positive index $t \in\{1, \ldots, T\}$. Such as $T=I-1$. In this view we can calculate $Z_{t}^{0}, \widehat{R}_{t}$ of each calender year and the sum of all payments of the development lozenge will give the ultimate claims 
amount $\widetilde{Z}$. We can complete the right part of the lozenge (future part) of the incremental payments by,

$$
\mathbb{E}\left(X_{i, t+1} \mid X_{i, i-I}, \ldots, X_{i, t}\right)=S_{i, t} \cdot\left(f_{-i+t+1}-1\right) .
$$

\subsection{Estimation of parameters in the calendar years view}

\subsubsection{The development factors at time $t$}

For each development year $t \in\{-T, \ldots,-1\}$, the development factors (Chain ladder factors) are estimated by

$$
\widehat{f_{t}}=\frac{\sum_{i=1}^{-t} X_{i, i+t}}{\sum_{i=1}^{-t} S_{i, i+t-1}}+1, \quad \text { where } S_{i, t}=\sum_{l=i-I}^{t} X_{i, l} .
$$

We observe that the development factors are indexed by the calendar year $(t)$, but they calculate always the proportion between the development years.

\subsubsection{The past obligations at time $t$}

The process of past obligations for each $t \in\{-T, \ldots, 0\}$, i.e the past claims amount $X_{i, j}$, has already been paid for each calendar year till the current date $t=0$, which is defined by

$$
Z_{t}^{0}=\sum_{i=1}^{t+I} X_{i, t} .
$$

We can obtain the overall past obligations by

$$
Z^{0}=\sum_{t=-T}^{0} Z_{t}^{0} .
$$

\subsubsection{Claims reserves at time $t$}

To obtain the estimators of the outstanding claims reserves for each calendar year $t$, we denote for $t \in\{1, \ldots, T\}$

$$
\widehat{R}_{t}=\sum_{i=t+1}^{I} \widehat{X}_{i, t} .
$$

also the reserves for each year $i$, for $i \in\{2, \ldots, I\}$

$$
\widehat{R}_{i}=\sum_{t=1}^{i-1} \widehat{X}_{i, t} .
$$


and their sum will give the overall reserves

$$
\widehat{R}=\sum_{t=1}^{T} \widehat{R}_{t}=\sum_{i=2}^{I} \widehat{R}_{i} .
$$

\subsubsection{The ultimate claims amount}

We can calculate the yearly ultimate claims amount by

$$
\widehat{\widetilde{Z}}_{i}=\sum_{t=-T+i-1}^{0} X_{i, t}+\sum_{t=1}^{i-1} \widehat{X}_{i, t}
$$

The process $\widehat{\widetilde{Z}}$ defined by

$$
\widehat{\widetilde{Z}}=\sum_{i=1}^{I} \widehat{\widetilde{Z}}_{i}=\sum_{t=-T}^{0} Z_{t}^{0}+\sum_{t=1}^{T} \widehat{R}_{t} .
$$

\subsection{Claim development result in the calendar years view}

We can apply the same definitions of the CDR presented above to get the observable $\widehat{C D R_{i}}(I+1)$

$$
\widehat{C D R}_{i}(I+1)=\widehat{R}_{i}^{D_{i, t}^{I}}-\left(X_{i, 1}+\widehat{R}_{i}^{D_{i, t}^{I+1}}\right)=\widehat{\widetilde{Z}}_{i}^{I}-\widehat{\widetilde{Z}}_{i}^{I+1}
$$

Where $X_{i, 1}$ is simulated by a one year bootstrapping, with $X_{1,1}=0$.

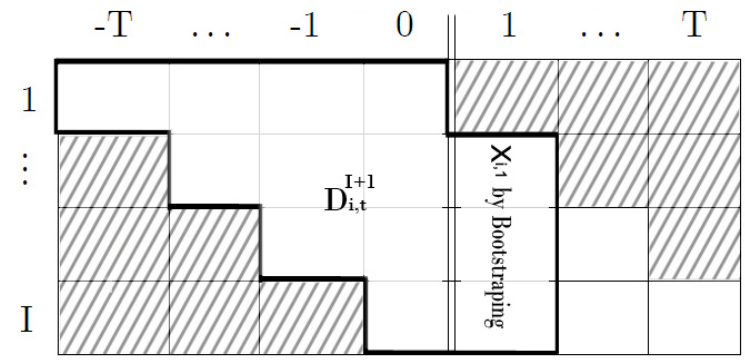

Figure 6: Claim Development Result in the calendar years view

The development factors

$$
\widehat{f}_{t}^{I}=\widehat{f}_{t} \quad \text { and } \quad \widehat{f}_{t}^{I+1}=\frac{\sum_{i=1}^{-t+1} X_{i, i+t}}{\sum_{i=1}^{-t+1} S_{i, i+t-1}}+1 .
$$


The ultimate claims amount

$$
\widehat{\widetilde{Z}}_{i}^{I}=\widehat{\widetilde{Z}}_{i} \text { and } \quad \widehat{\widetilde{Z}}_{i}^{I+1}=\sum_{t=-T+i-1}^{1} X_{i, t}+\sum_{t=2}^{i-1} \widehat{X}_{i, t} .
$$

Yearly claims reserves

$$
\widehat{R}_{i}^{D_{i, j}^{I}}=\widehat{R}_{i}, \quad \text { and } \quad \widehat{R}_{i}^{D_{i, j}^{I+1}}=\sum_{t=2}^{i-1} \widehat{X}_{i, t},
$$

The aggregate $C D R$ is given by

$$
\widehat{C D R}(I+1)=\widehat{\widetilde{Z}}^{I}-\widehat{\widetilde{Z}}^{I+1}=\sum_{i=2}^{I} \widehat{C D R}_{i}(I+1)
$$

\section{Numerical example and conclusions}

For our numerical example we use the data set given in Figure 7. The table contains incremental payments for accident years $i \in\{1, \ldots, 9\}$. We will apply our approach (Stochastic Chain ladder using incremental payments) on the incremental payments data $X_{i, j}$, we first calculate the development factors

\begin{tabular}{|c|c|c|c|c|c|c|c|c|c|c|c|c|}
\hline \multirow[b]{2}{*}{ Accident year i } & \multicolumn{9}{|c|}{ Development year j } & \multirow{2}{*}{$\begin{array}{c}\text { Reserve } \\
\hat{R}_{i}\end{array}$} & \multirow{2}{*}{$\begin{array}{c}\text { Ultimate } \\
\widehat{\widetilde{Z}}_{i}\end{array}$} & \\
\hline & 1 & 2 & 3 & 4 & 5 & 6 & 7 & 8 & 9 & & & \\
\hline 1 & 2202584 & 1007865 & 257673 & 76948 & 76557 & 23009 & 24376 & 5499 & 4122 & 0 & 3678633 & \\
\hline 2 & 2350650 & 1202373 & 230823 & 56221 & 25120 & 13557 & 19537 & 4144 & 4378 & 4378 & 3906803 & \\
\hline 3 & 2321885 & 1102305 & 276686 & 97322 & 56557 & 24238 & 19832 & 4968 & 4379 & 9347 & 3908172 & \\
\hline 4 & 2171487 & 993787 & 230567 & 70612 & 49250 & 32718 & 19837 & 4547 & 4008 & 28392 & 3576813 & \\
\hline 5 & 2140328 & 1016751 & 242183 & 101258 & 85292 & 22572 & 20173 & 4624 & 4076 & 51444 & 3637256 & \\
\hline 6 & 2290664 & 1047533 & 212135 & 90704 & 58732 & 23289 & 20814 & 4771 & 4205 & 111811 & 3752847 & \\
\hline 7 & 2148216 & 1071559 & 208560 & 79368 & 56581 & 22436 & 20052 & 4596 & 4051 & 187084 & 3615419 & \\
\hline 8 & 2143728 & 1014853 & 227107 & 78380 & 55878 & 22157 & 19802 & 4539 & 4001 & 411864 & 3570445 & \\
\hline 9 & 2144738 & 1020741 & 227603 & 78551 & 56000 & 22205 & 19845 & 4549 & 4010 & 1433505 & 3578243 & \\
\hline & & & & & & & & & & 2237825 & 33224631 & Total \\
\hline$f_{\mathrm{j}}$ & 1,4759 & 1,0719 & 1,0232 & 1,0161 & 1,0063 & 1,0056 & 1,0013 & 1,0011 & & & & \\
\hline
\end{tabular}
according to formula (1) then we complete the lower triangle, and finally we obtain the amounts we seek to put in reserve.

Figure 7: Run-off triangle (incremental payments, in Euro 1000) for time $I=9$

The results are identical to those given by the classic Chain ladder using cumulated payments, so we can calculate our reserves simply without need to calculate the cumulated triangle, by avoiding a step of calculation and we can got a clear image about information we have in the triangle, and the formula we use here are more easy by applying simple summations. Let's move at present to the new tabulation form for the calendar years view and we can recalculate the reserves as in figure 8 . 


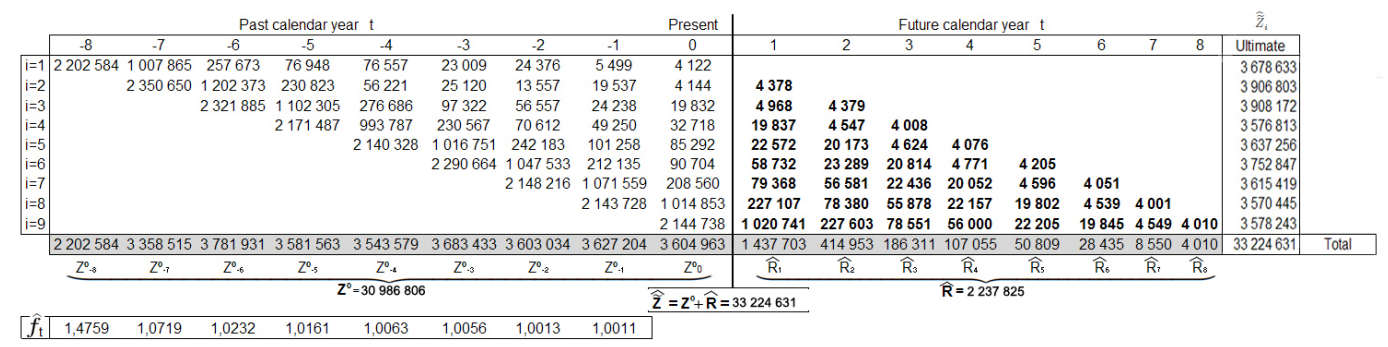

Figure 8: Run-off triangle in calendar view (incremental payments, in Euro 1000) for time $\mathrm{I}=9$

We observe here that we can calculate reserves of each calendar year (which is represented by diagonals in classic triangle) by calculating first the development factors for each calendar year t using formula (21), then we can find the overall reserve. By using the new tabulation form we find the same development factors, also we got identical overall reserves and ultimate claims amounts comparing with those calculated by our incremental approach (stochastic chain ladder using incremental payments). Now we will calculate $C D R$ for the two cases ( incremental approach and for calendar year view), the results are given in figure 9 .

\begin{tabular}{c|ccc}
\hline & $\hat{\widetilde{Z}}_{i}^{I}$ & $\widehat{\widetilde{Z}}_{i}^{I+1}$ & $\widehat{C D R}_{i}(I+1)$ \\
\hline 1 & 3678633 & 3678633 & 0 \\
2 & 3906803 & 3906699 & 104 \\
3 & 3908172 & 3906113 & 2059 \\
4 & 3576813 & 3572156 & 4657 \\
5 & 3637256 & 3635610 & 1646 \\
6 & 3752847 & 3771609 & -18762 \\
7 & 3615419 & 3641266 & -25847 \\
8 & 3570445 & 3575394 & -4949 \\
9 & 3578243 & 3566649 & 11594 \\
\hline Total & 33224631 & 33254129 & -29498 \\
\hline \hline
\end{tabular}

Figure 9: Realization of the observable CDR at time $t=I+1$, in Euro 1000

\subsection{Conclusion}

This work has examined new ways to estimate the amounts place in reserve to perform in the future payments related to claims incurred.

First, the calculation of claims reserves using a stochastic incremental approach by modifying Mack's model, so we establish the require formulae using 
only incremental payments which avoid calculation of the cumulative triangle, and we can got identical results see Comparison [2.4]. We calculate the CDR for this case, and we apply the bootstrap by developed a new formula see (19) which avoid 3 steps in calculations.

Second, we examined the incremental approach in calendar year view, so we propose a new form of tabulation Figure 5, so we can easily calculate the past obligation of each calendar years ( $t$ ), the claims reserves of each accident year (i) and the overall reserve.

The incremental approach give us the advantage to avoid lot of steps in different cases, and we can observe the simplicity of the formulae used to give identical results.

\section{References}

[1] M. Buchwalder, H. Buhlmann, M. Merz and M. Wuthrich, Legal Valuation Portfolio in Non-Life Insurance, Conference paper presented at the 36th Int. ASTIN Colloquim ETH Zurich, (2005).

[2] K.Th. Eisele and Ph. Artzner, Multiperiod Insurance Supervision: TopDown Models, European Actuarial Journal, 1 (2011), 107 - 130.

[3] P. D. England and R. J. Verrall, Stochastic Claims Reserving in General Insurance, Presented to the Institute of Actuaries, (2002).

[4] T. Mack, Measuring the Variability of Chain Ladder Reserve Estimates, Casualty Actuarial Society Forum, (1994), 101 - 182.

[5] T. Mack, Distribution-free calculation of the standard error of chainladder reserve estimates, ASTIN Bulletin, 23:2 (1993), 213 - 225.

[6] M. Merz, M. Wuthrich and N. Lysenko, Uncertainty of the Claims Development Result in the Chain Ladder Method, Scandinavian Actuarial Journal, 1 (2009), 63 - 84.

[7] C. Partrat, N. Pey and J. Schilling, Delta Method and Reserving, ASTIN Colloquium, Zurich, Switzerland, (2005).

\section{Received: October 23, 2012}

